\title{
Elevated serum and urine levels of progranulin (PGRN) as a predictor of microglia activation in the early phase of traumatic brain injury: a further link with the development of neurodegenerative diseases
}

\author{
Mieszko Olczak ${ }^{1}$, Łukasz A. Poniatowski ${ }^{2,3}$, Agnieszka Siwińska ${ }^{1}$, Magdalena Kwiatkowska $^{1}$, Dominik Chutorański ${ }^{4}$, \\ Teresa Wierzba-Bobrowicz ${ }^{4}$ \\ ${ }^{1}$ Department of Forensic Medicine, Centre for Biostructure Research, Medical University of Warsaw, Poland, ${ }^{2}$ Department of \\ Experimental and Clinical Pharmacology, Centre for Preclinical Research and Technology (CePT), Medical University of Warsaw, \\ Poland, ${ }^{3}$ Department of Neurosurgery, Maria Skłodowska-Curie National Research Institute of Oncology, Warsaw, Poland, \\ ${ }^{4}$ Department of Neuropathology, Institute of Psychiatry and Neurology, Warsaw, Poland
}

\begin{abstract}
Traumatic brain injury (TBI) is a frequent finding during forensic autopsies and neuropathological examinations in medico-legal practices. Despite the unprecedented attention currently focused on TBI pathogenesis, there is a need to improve its diagnostics through the use of novel biomarkers to facilitate detection, treatment, and prognosis. Recently, growth factor progranulin (PGRN) has attracted significant attention because of its neurotrophic and anti-inflammatory activities. The role of PGRN in TBI has not been widely discussed, although PGRN-related neuroinflammatory and neurodegenerative phenomena have been described. The aim of this study was to identify PGRN concentration levels in biofluids and examine PGRN and CD68 protein expression in brain tissue using immunohistochemical staining in individuals with fatal TBI in its early phase. The study was performed using cases $(n=30)$ of fatal head injury and control cases $(n=30)$ of sudden death. The serum and urine were collected within $\sim 24 \mathrm{~h}$ after death and compared using the ELISA test, where brain specimens were stained with anti-PGRN and anti-CD68 antibodies. In our study, we observed elevated concentration levels of PGRN in the serum and urine of TBI individuals in the early phase of TBI. These changes were accompanied by increased expression of PGRN in the frontal cortex ( $1^{\text {st }}-3^{\text {rd }}$ layers), in which antiCD68 immunostaining revealed disseminated cortical microglia activation. The possible implementation of performing such assays offers a novel and interesting tool for investigation and research regarding TBI diagnosis and pathogenesis. Furthermore, the above-mentioned surrogate biofluid assays may be useful in clinical prognosis and risk calculation of non-fatal cases of TBI, considering the development of neurodegenerative conditions of TBI individuals.
\end{abstract}

Key words: progranulin, traumatic brain injury, microglia, serum, urine, neurodegenerative diseases.

\section{Introduction}

According to data provided by the United States (US) Centers for Disease Control and Prevention
(CDC), surveillance studies on traumatic brain injury (TBI) for the year 2013 show an average of 2.5 million TBI-related emergency department (ED) visits in

Communicating author:

Łukasz A. Poniatowski, MD, Department of Experimental and Clinical Pharmacology, Centre for Preclinical Research and Technology (CePT), Medical University of Warsaw, 1B Banacha St., 02-097 Warsaw, Poland, e-mail: lukasz.poniatowski@gmail.com 
the US, resulting in over 282,000 related hospitalisations and 56,000 deaths [46]. In Europe, according to 28 epidemiological studies from 16 countries, the yearly incidence of TBI is estimated at 262 per 100,000 people, with an average mortality rate of 10.5 per 100,000 people [37]. Standardised population data from 24 countries provided by Eurostat (European Statistical Office) in 2012 suggest that the pooled age-adjusted incidence of TBI was 287.2 per 100,000 people, with an average mortality of 11.7 per 100,000 [29]. Insights afforded by detailed studies and current understanding of key pathological processes in TBI pathogenesis suggest that this condition should be considered as a long-lasting complex neurodegenerative disease and not only as a single pathophysiological phenomenon [19]. The complexity of the natural course of TBI-related pathophysiological events occurs as a result of 2 subsequent and not clearly demarcated phases i.e. primary and secondary (delayed) injury - where virtually all parts and compartments of the brain are vulnerable and could be affected, which represents a distinct biology [21]. Primary injury is categorised as the rapid and irreversible result of a transient application ( $20 \mathrm{msec})$ of various external physical forces the character of which determines the severity of the initial injury [28]. In contrast, secondary injury develops over time as a non-linear phenomenon ranging in scale from minutes to years, and includes numerous self-propagating synergistic cellular, molecular, neurometabolic, and neurochemical events acting as a neurodegenerative effect initiated by the primary mechanical force [9]. TBI is not only related to local neural damage but is a multi-system pathology, which is revealed in its multicomplex systemic immune response via a broad spectrum of phenomena including changes in transcriptome set, proteome set, immune cell recruitment, and production of inflammatory mediators [20]. Neuroinflammation is known to be one of the key emerging front-line secondary injury components that drive ongoing neuronal injury [56]. One of the main multiphasic events in $\mathrm{TBI}$ and spinal cord injury $(\mathrm{SCl})$ is the local activation and proliferation of native microglia (Iba-1+) and astrocytes (GFAP+) associated with an overflow of central nervous system (CNS) by peripheral immune cells recruited in a time-dependent manner, which participate together in the creation of a tightly regulated inflammatory microenvironment [7]. Activated microglia and astrocytes serve in this case as one of the main sources of cytokines, proteinases, extracellular matrix (ECM) molecules, and growth factors in the lesion epicentre [24,27]. In regard to scientific and clinical progress, there is a continuing need to attempt to establish novel relevant bioassays and compilation platforms for use in the field of TBI diagnostics in both in ante- and post-mortem cases [10]. Currently available biofluid assays for TBI in the pre- and clinical settings include a panel of neuronal, astroglia, and axonal injury indicators, which can be detected mainly in cerebrospinal fluid (CSF) and serum [14]. Recently, multifunctional and pleiotropic growth factor progranulin (PGRN) has attracted significant attention in the neuroscience community, because of its potent and specific neurotrophic, anti-inflammatory, and immunomodulatory activities [54]. Two breakthrough studies published in the year 2006 revealed that null mutations and haploinsufficiency in the PGRN gene were identified as a cause of several familiar forms of frontotemporal lobar degeneration (FTLD) and sparked landmark research aimed at elucidating PGRN function in CNS $[4,13]$. The ultrastructure of PGRN covers a polypeptide chain composed of 593 amino acid residues with a total molecular weight of $68.5 \mathrm{kDa}$, becoming $88 \mathrm{kDa}$ when secreted from cells after glycosylation [5]. In this case, the polypeptide chain of PGRN contains 7.5 tandem repeats of a highly conserved 12 cysteines (Cys) rich motif (CX5-6CX5CCX8CCX6CCXDX2HCCPX4CX5-6C) in the order P-G-F-B-A-C-D-E (p-P1-G-P2-F-P3-B-P4-A-P5-C-P6-D-P7-E), where A-G are full repeats and $p$ is one half-motif [47]. Within the pathologies of the nervous system, dysregulation of PGRN functioning associated with mutations in its encoding gene have been to date potentially linked with the development of Parkinson's disease, Alzheimer's disease, amyotrophic lateral sclerosis, motor neuron disease, neuronal ceroid lipofuscinosis, Creutzfeldt-Jakob disease, epilepsy, bipolar disorder, and schizophrenia [12]. In the brain, PGRN is predominantly expressed in pyramidal neurons and activated microglia, whereas astrocytes have been shown to take up PGRN and serve as a reservoir $[2,38]$. In a wider context, it seems that PGRN serves as a physiological regulator of homeostasis and neuronal functions, a neurotrophic factor which regulates neurite outgrowth and maintains its survival [48]. Furthermore, new evidence indicates that PGRN may function as a pivotal chemoattractant by recruiting and shift-activating microglia and mac- 
rophages [18]. The growing knowledge about PGRN and its associated multiple receptors and pathways suggest that in several pathologies PGRN could constitute a distinct, critical molecule essential to the biological functioning of all cells in the nervous system. Studies published over the last several years describing the newly discovered phenomena in regard to the competitive influence of endogenous PGRN and related smaller polypeptides on receptors associated with tumour necrosis factor $\alpha$ (TNF- $\alpha$ )/ tumour necrosis factor receptor $1 / 2$ (TNFR1/2) may indicate the main potential mechanism of PGRN contribution in pathophysiological mechanisms that precisely orchestrate and modulate neuroinflammatory phenomena $[1,30]$. To date, the role of PGRN and its potential application as a biomarker has not been widely discussed in the context of TBI, although currently available literature describes PGRN-related neuroinflammatory and neurodegenerative phenomena, well studied in preclinical rodent models. In order to provide an accurate and current overview of the issue, we have performed a study in an attempt to elucidate and identify if elevated PGRN concentration levels in biofluids such as blood and urine are seen in cases of TBI in a population-based autopsy screening. We have also extended our study by the comparative examination of the PGRN and CD68 protein expression in the obtained brain tissue by performing additional immunohistochemical and histological staining. Finally, we discuss the possible role of PGRN in TBI diagnostics in both ante- and post-mortem case settings, with a special focus paid to relevant clinical implications, aiming to delineate its potential predictive role in both survival outcome and development of post-traumatic neurodegenerative conditions.

\section{Material and methods}

\section{Autopsy cases}

The study and following analyses have been conducted on biofluids and tissue samples collected during routine autopsies carried out by forensic pathologists from the Department of Forensic Medicine at the Medical University of Warsaw. In this case, a total of 60 cases $(n=60)$ was included in this study and subsequently divided into 2 consecutive groups. Consequently, the study group consisted of 30 cases $(n=30)$ of focal and severe head injury, which was confirmed to be the cause of death by the forensic autopsy. The control group included 30 cases $(n=30)$ of instantaneous death due to cardiopulmonary failure (cardiac arrest) without injuries of the head and morphological signs of a brain injury on a macroscopic level. Both groups consisted of cases that were not followed by cardiopulmonary resuscitation (CPR), submission to hospital, and did not subsequently undergo any other emergency or intensive therapy procedures (death at the place of the incident). After death was confirmed, the corpses were transported to cold storage with controlled temperature conditions $\left(4^{\circ} \mathrm{C}\right)$, where they were kept until the forensic autopsy was performed and material was collected for examination. The supplemental data regarding the circumstances of the death was acquired from the available case files during the medico-legal investigation. Additional medical records and family testimony underwent a detailed qualification process based on specified exclusion criteria. Exclusion criteria included neurological diseases: frontotemporal lobar degeneration, Alzheimer's disease, Parkinson's disease, amyotrophic lateral sclerosis, motor neuron disease, neuronal ceroid lipofuscinosis, multiple sclerosis, epilepsy, cerebrovascular disease, Creutzfeldt-Jakob disease, schizophrenia, and bipolar disease; and non-neurological diseases: rheumatoid diseases, other inflammatory and autoimmune diseases, diabetes mellitus, impaired kidney function and disease, impaired liver function and disease, previous history of malignancy (tumour), history of infectious disease within 6 months, and history of acute trauma or surgery within 6 months. Implemented strict selection was performed with the assumption that changes in PGRN presence and concentration level are known to be related to a variety of neurological and non-neurological human disorders. The average age of the deceased was $48.1 \pm 9.7$ years in the study group and $52.4 \pm 11.2$ years in the control group. We did not observe statistical $(p>0.05)$ differences regarding the lifetime between groups.

\section{Obtaining and preparing biofluid and tissue specimens}

The medico-legal autopsy procedure was carried out within 12-24 $\mathrm{h}$ after death, during which 2 types of material for subsequent analyses and examinations were collected. The primary type consisted of biofluid samples including blood and urine, which 
were obtained for assessment of PGRN occurrence and subsequent concentration level. Another type of tissue material were specimens of the frontal lobe of the brain, consisting of the corpus callosum and cingulate cortex, used for H\&E and immunohistochemical stainings. Blood samples were obtained through femoral vein puncture, whereas urine samples were aspirated directly from the bladder by a percutaneous suprapubic puncture after decontamination of the skin with a $90 \%$ ethanol $\left(\mathrm{C}_{2} \mathrm{H}_{5} \mathrm{OH}\right)$ solution prior to both procedures. In both cases, the remaining fluid containing particulate material was not used during sampling, to avoid potential contamination of the material. Blood samples were immediately centrifuged at $6000 \mathrm{rpm}$ for $10 \mathrm{~min}$ to obtain serum, whereas urine samples were processed at $1600 \mathrm{rpm}$ for $10 \mathrm{~min}$ to obtain a supernatant. Both sample types were stored in sterile sample tubes and preserved $\left(\right.$ at $-80^{\circ} \mathrm{C}$ ) until further analysis. All samples with features of haemolysis were excluded from testing. The brain tissue fragments taken from frontal lobes were collected in a routine way and then fixed in $10 \%$ buffered formalin $\left(\mathrm{CH}_{2} \mathrm{O}\right)$ and subsequently embedded in paraffin wax $\left(\mathrm{CnH}_{2 n+2}\right)$ blocks.

\section{ELISA}

Analysis of the occurrence and concentration level of PGRN in sampled biofluids such as serum and urine was performed using an enzyme-linked immunosorbent assay (ELISA) test. A series of assays were carried out using the commercially available Human PGRN (Progranulin) ELISA Kit (EH4861; Wuhan Fine Biotech, Wuhan, Hubei, China) according to the standardised manual supplied by the manufacturer. The following series of assays was tested in 2 subsequent repetitions, and average results were used for analysis.

\section{Immunohistochemical and histological methods}

The collected intact brain specimens were stained histologically with haematoxylin and eosin $(H \& E)$ as well as immunohistochemically using assigned antibodies. In this case, the brain slices were consecutively stained with anti-Granulin antibody (FNab03632; Wuhan Fine Biotech, Wuhan, Hubei, China) in $1: 100$ dilution and anti-CD68 antibody (M0814; Dako, Glostrup, Denmark) in $1: 75$ dilution according to the standardised manual sup- plied by the manufacturers. The microphotographs of the brain slices were taken using an Olympus BX53 microscope (Olympus Optical, Tokyo, Japan) equipped with an Olympus UC90 digital camera (Olympus Optical, Tokyo, Japan) and coupled with on-screen data acquisition and image analysis cellSens Dimension version 2.3 software (Olympus Soft Imaging Solutions, Münster, Germany).

\section{Statistical analysis}

The data were analysed, and comparisons of both groups were performed using Statistica 13.1 PL (StatSoft, Tulsa, OK, USA) software package for Windows (Microsoft Corporation). The results were considered statistically significant when $p$-values were less than $0.05(p<0.05)$. The one-sample Kolmogorov-Smirnov test was used to assess whether the PGRN concentration levels were normally distributed. Because the concentrations of PGRN in serum and urine were not normally distributed, the data were analysed using a Mann-Whitney-Wilcoxon (MWW) rank-sum test (two-tailed). The data were presented as a mean value \pm SEM.

\section{Results}

\section{Concentration of PGRN in biofluids}

The performed ELISA test revealed increased PGRN concentration levels in both the serum and urine of the study group, as compared to the control group. The results presented in Figure 1A show an elevated serum concentration of PGRN in the study group $(51.97 \pm 11.01 \mathrm{ng} / \mathrm{ml})$ compared to the control group (20.62 $\pm 2.81 \mathrm{ng} / \mathrm{ml})$, which was statistically significant $(p<0.0004)$. Consecutively, Figure 1B shows an elevated urine concentration of PGRN in the study group $(4.96 \pm 0.3 \mathrm{ng} / \mathrm{ml})$ compared to the control group (3.04 $\pm 0.42 \mathrm{ng} / \mathrm{ml})$, which was also statistically significant $(p<0.02)$.

\section{Immunohistochemical and histological stains}

The neuropathological examination revealed macroscopic signs of a lethal brain injury (brain contusion and laceration, subdural haemorrhage, intraventricular bleeding) assumed as the cause of death in all cases from the study group. Control group cases revealed no morphological signs of a brain injury either on the macro- or microscopic levels. The microscopic assess- 
A

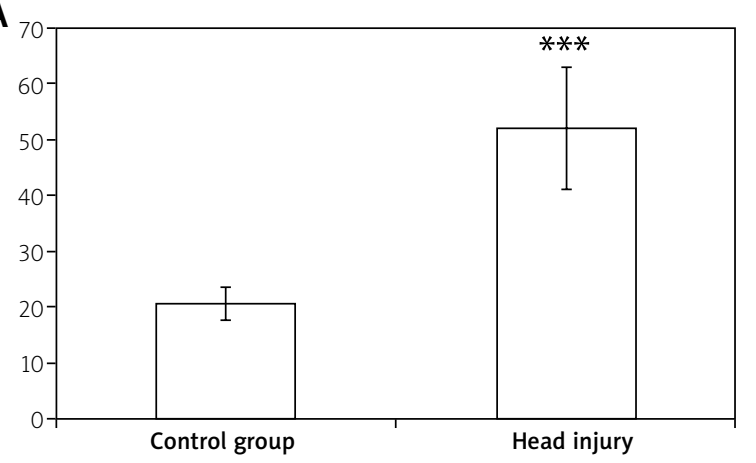

B

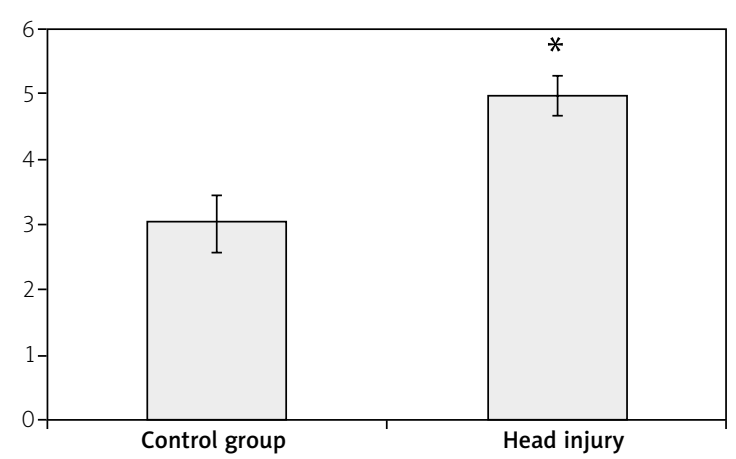

Fig. 1. The proportion of PGRN concentration levels in obtained biofluids such as blood serum (A) and urine (B) measured in $\mathrm{ng} / \mathrm{ml}$ respectively. Elevated PGRN concentration levels are visible in the serum and urine in the study group. Data are presented as a mean value \pm SEM. ${ }^{*}$ Differs from control group, ${ }^{*} p<0.05,{ }^{* * *} p<0.001$.
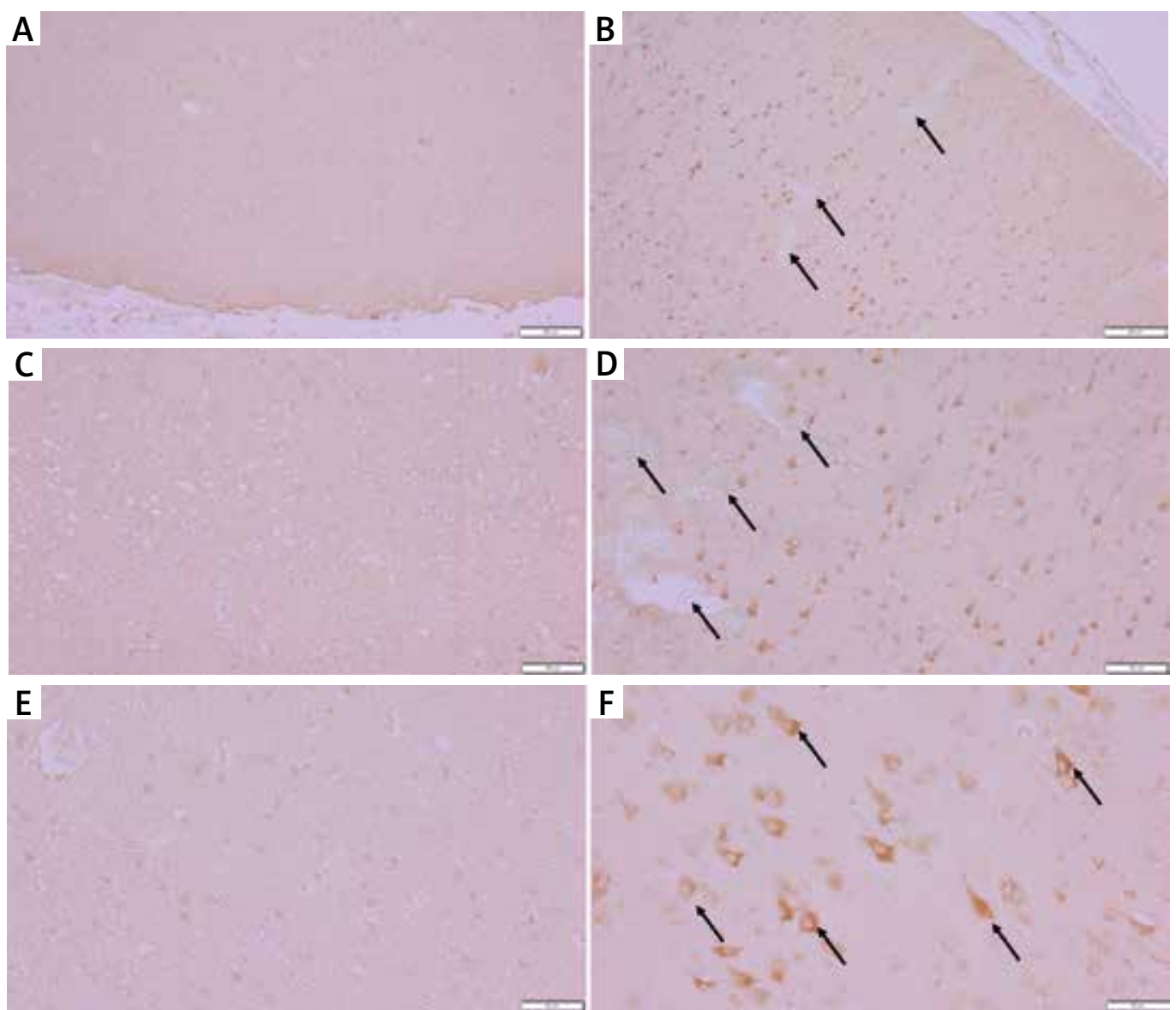

Fig. 2. Representative microphotographs showing PGRN expression in examined brain slices of frontal cortex. A) Control group, minimal PGRN expression (100x), B) Study group, increased PGRN expression in neurons, brain contusions marked with the black arrows (100x), C) Control group, minimal PGRN expression (200x), D) Study group, increased PGRN expression in neurons, brain contusions marked with the black arrows (200x), E) Control group, minimal PGRN expression (400x), F) Study group, increased PGRN expression in neurons marked with the black arrows (400x). 

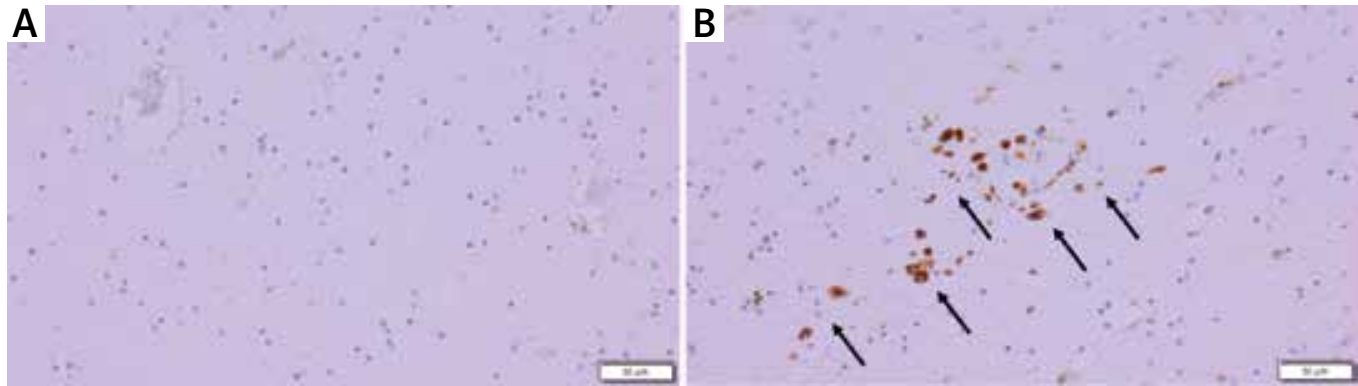

Fig. 3. Representative microphotographs showing microglia activation (anti-CD68 immunostaining) in examined brain slices of frontal cortex. A) Control group, no CD68 expression associated with microglia activation (400x), B) Study group, increased microglia activation marked with black arrows (400x).

ment did not show specific signs of neurodegeneration in obtained brain slices from both evaluated groups (data not shown). The performed anti-PGRN immunostaining revealed increased expression of the protein in the frontal cortex, especially in the $1^{\text {st }}-3^{\text {rd }}$ layers in the study group, as compared to the control group (Fig. 2). Consecutively, the anti-CD68 immunostaining revealed disseminated cortical microglia activation in the study group, as compared to the control group (Fig. 3).

\section{Discussion}

In the following study, we have consecutively shown statistically significant elevated serum and urine concentration levels of PGRN in the study group (severe head injury/TBI in its early phase), compared to the control group. These changes were accompanied by generalised microglia activation in the frontal cortex of the study (head injury) group. In healthy human individuals, the CSF concentration level of PGRN oscillates in the range $4.07-6.60 \mathrm{ng} / \mathrm{ml}$ $(\leq 5 \mathrm{ng} / \mathrm{ml})$ and is relatively weakly correlated with modifiers such as demographic and anthropometric parameters (including genetic factors) compared to its quantified serum equivalent value $[33,51]$. Therefore, the serum and CSF concentration of PGRN do not correlate, which indicates a differential regulation of its central and peripheral levels [50]. As stated in the methodology above, the strict case selection and qualification process based on neuropathological examination and specified exclusion criteria, which included the presence of conditions previously proposed and documented to be PGRN dependent (including lifetime), potentially influenced the obtained results. This indicates the very important issue that elevated PGRN serum and urine concen- tration levels occur in the case of the early TBI phase, and they are probably associated with the occurrence of axonal and neuronal damage, which is consistent with previous studies on acute neurological conditions, e.g. Xie et al. in 2016 observed increased PGRN serum concentration levels in patients with acute ischaemic stroke and provide an independent prognostic value for all-cause mortality [53]. Similar observations were observed in the study of LasekBal et al. in 2019, in which increased PGRN levels in serum were noticed on the first day after stroke [25]. Although the multidirectional actions of PGRN, including a variety of physiological and pathological processes within the nervous system, are well known, there are few available data on the role of PGRN in the pathogenesis of TBI. In this case, preclinical transgenic rodent studies using a complex set of proteomic, transcriptomic, and immunofluorescence methods provide the main insight into the implied role of PGRN in the pathophysiology of TBI. In the initial study evaluating this issue, Matzilevich et al. in 2002 for the first time observed that hippocampal (CA) expression of the PGRN gene is up-regulated after controlled cortical impact (CCI) to LongEvans rats [31]. A study with a similar methodology was conducted by Wang et al. in 2010 on C57BL/6 mice subjected to $\mathrm{CCl}$, which exposed a correlative microRNA-107 (miR-107) expression decrease within the hippocampus with an augmentation of PGRN expression [49]. Regarding the interconnected topic of neurotrauma, it is worth mentioning that similar findings were made in cases of $\mathrm{SCl}$ - Naphade et al. in 2010 observed more intense immunoreactivity of PGRN throughout the injury epicentre that peaked at 7-14 days in the C57BL/6 mice that were subjected to midthoracic (Th9 level) contusion [34]. Additionally, the coexisting PGRN immunoreactivity was asso- 
ciated with an increase of CD11b and CD68 marker immunoreactivity, indicating the involvement of primarily activated microglia and macrophages. Consecutively, the study of Tanaka et al. in 2013 performed on C57BL/6J mice that where PGRN-deficient (KO) indicates that PGRN expression increases after $\mathrm{TBI}$, and that CD68-positive activated microglia constitute a major source of PGRN [45]. Simultaneously, PGRN deficiency was associated with exacerbated inflammatory response observed as an increased proportion of CD68-positive activated microglia where no changes in CD11b immunoreactivity were observed. After the first evaluation of this topic, we have shown a similar increase of PGRN and CD68 immunoreactivity within contused brains. In the adult brain, PGRN is constitutively expressed within all 6 layers of the neocortex, remaining strongest in the $5^{\text {th }} 6^{\text {th }}$ layer comprising pyramidal and multiform neurons; whereas, in the cases of TBI evaluated in our study, it is highly expressed within the $1^{\text {st }}-3^{\text {rd }}$ layers after trauma $[8,15,16]$. In our study the head injury group presented disseminated microglia activation in the examined brain slices of the frontal cortex. Therefore, the elevated PGRN concentration level in serum can be linked with the activation of microglia associated with its proliferation, aggregation, and further differentiation. Similar findings of disseminated microglia activation in the early phase of TBI were observed in our previous study [36]. Until now, only a few investigations have considered issues relating to the influx of brain-originated proteins into the urinary and renal system in neurological diseases [3]. Therefore, to date, the assay of potential variations due to neurological diseases did not take into consideration the "outlying" anatomical relation between the urinary and nervous systems, along with the existence of restricted and periodic barrier access $[3,55]$. Detection and assay of PGRN concentration in urine did not find much of interest regarding the scientific and clinical field. In this case, its usefulness was evaluated in the case of diabetes, cancer, and rheumatoid diseases $[35,43,52]$. Nevertheless, the combined re-evaluation of both issues provided us with new information on the potential PGRN efflux from the injured brain into peripheral biofluid such as evaluated urine. In this way, for the first time, it was shown that evaluation of the PGRN concentration could constitute a novel assay suitable for post-mortem axonal injury assessment when used alone or in combination with other diagnostic tools, which overall could have the potential to contribute to early TBI diagnosis. In addition to the above-described usefulness of PGRN assays in post-mortem diagnostics of TBI, special consideration should be additionally given to patients who survive and recover after brain trauma. Evaluation and validation of different PGRN gene-associated variants showed that patients carrying PGRN mutations were reported to exhibit decreased plasma levels of PGRN compared to individuals without mutations, suggesting the possibility of a loss-of-function disease mechanism [11]. As was previously reported, TBI is an environmental risk factor of FTLD - according to retrospective case-control analyses, patients with diagnosed FTLD were $\sim 3.3$ (range, 1.3-8.1) times more likely to have experienced head trauma as compared to appropriate control groups [22,42]. Insights afforded in our study seem to correspond with the abovementioned relationship and indicate the significance of TBI in patients suffering in the future from neurodegenerative diseases. Recently, PGRN was suggested to participate in neuronal recovery within the CNS as a neurotrophic and anti-inflammatory factor [41]. In the study of Menzel et al. in 2017, PGRN-deficient mice that underwent $\mathrm{CCl}$ displayed increased perilesional axonal injury, which was associated with increased transcription of inflammatory and decreased transcription of anti-inflammatory cytokines [32]. Similarly, intracerebroventricular administration of PGRN protein before $\mathrm{CCl}$ was associated with the reduction of brain damage and functional deficit, as well as restoration of cytokine transcription profile with concomitant reduction of astrogliosis. The related functions of PGRN are associated with its metabolism related with its proteolytic cleavage, leading to the release of several individually liberated GRN included in 56-57 aa residues - which, after proteolysis occurs in both, in individual or linked combination form $(\sim 6-25 \mathrm{kDa})$, have opposing inflammatory properties $[6,39]$. The proteolytic cleavage process is mediated by various intra- and extracellular serine (Ser) and threonine (Thr) proteinases; these interactions occur in both directions based on a feed-forward loop $[44,54,57]$. In this case, the initial trauma seems to be associated with a disruption of PGRN metabolism, causing its insufficiency through an efflux of proteinases by microglia, all as part of an ongoing secondary injury mechanism associated with an increase of susceptibility to the develop- 
ment of neurodegenerative conditions even after a mild episode of TBI. Despite the obtained data, the role of PGRN in the pathogenesis of TBI and neurodegenerative diseases is multifactorial and is associated with multiple overlapping molecular issues. Our neuropathological observations using selected immunohistochemical and histological stains support the point of view that efflux of PGRN to several biofluids occurs and is associated with disseminated cortical microglia activation as an effect of ongoing neuroinflammatory reaction. Along these lines, the possible implementation of performed assays provides technical options for a novel and interesting tool for subsequent TBI research, diagnosis, and pathogenesis. Furthermore, the above-mentioned surrogate biofluid assays may be useful in clinical prognosis and risk calculation considering the development of neurodegenerative conditions of TBI individuals. Studies conducted over the past several years have indicated that assessment of serum molecular biomarkers are worthy of attention in neuroscientific practice, due to their non-invasiveness and cost-effectiveness to collect while bringing far-reaching information associated with the clinical status of the patient and predictions for disease forecasting $[17,40]$. At present, this outlook supports the implementation of not just single, but multiplexed platforms of less invasive and rapid assays in various neurological conditions covering diagnosis and monitoring $[23,26]$.

\section{Acknowledgments}

This study was supported by internal grant $1 \mathrm{MB} / \mathrm{PM} 1 / 18$ conducted in the years 2018-2019 from the Medical University of Warsaw. The project was implemented with infrastructure financed by the European Union (EU) - The European Regional Development Fund (ERDF) within the "Infrastructure and Environment" operational program for the years 2007-2013. No additional external funding or support was received for this study.

\section{Disclosure}

The authors report no conflict of interest.

\section{References}

1. Ahmed Z, Mackenzie IR, Hutton ML, Dickson DW. Progranulin in frontotemporal lobar degeneration and neuroinflammation. J Neuroinflammation 2007; 4: 7.
2. Almeida S, Zhou L, Gao FB. Progranulin, a glycoprotein deficient in frontotemporal dementia, is a novel substrate of several protein disulfide isomerase family proteins. PLoS One 2011; 6 : e26454.

3. An M, Gao Y. Urinary biomarkers of brain diseases. Genomics Proteomics Bioinformatics 2015; 13: 345-354.

4. Baker M, Mackenzie IR, Pickering-Brown SM, Gass J, Rademakers R, Lindholm C, Snowden J, Adamson J, Sadovnick AD, Rollinson S, Cannon A, Dwosh E, Neary D, Melquist S, Richardson A, Dickson D, Berger Z, Eriksen J, Robinson T, Zehr C, Dickey CA, Crook R, McGowan E, Mann D, Boeve B, Feldman H, Hutton M. Mutations in progranulin cause tau-negative frontotemporal dementia linked to chromosome 17. Nature 2006; 442: 916-919.

5. Bateman A, Belcourt D, Bennett H, Lazure C, Solomon S. Granulins, a novel class of peptide from leukocytes. Biochem Biophys Res Commun 1990; 173: 1161-1168.

6. Bateman A, Bennett HP. Granulins: the structure and function of an emerging family of growth factors. J Endocrinol 1998; 158: 145-151.

7. Beck KD, Nguyen HX, Galvan MD, Salazar DL, Woodruff TM, Anderson AJ. Quantitative analysis of cellular inflammation after traumatic spinal cord injury: evidence for a multiphasic inflammatory response in the acute to chronic environment. Brain 2010; 133: 433-447.

8. Bhandari V, Giaid A, Bateman A. The complementary deoxyribonucleic acid sequence, tissue distribution, and cellular localization of the rat granulin precursor. Endocrinology 1993; 133: 2682-2689.

9. Borgens RB, Liu-Snyder P. Understanding secondary injury. Q Rev Biol 2012; 87: 89-127.

10. Carpenter KL, Czosnyka M, Jalloh I, Newcombe VF, Helmy A, Shannon RJ, Budohoski KP, Kolias AG, Kirkpatrick PJ, Carpenter TA, Menon DK, Hutchinson PJ. Systemic, local, and imaging biomarkers of brain injury: more needed, and better use of those already established? Front Neurol 2015; 6: 26.

11. Chen-Plotkin AS, Martinez-Lage M, Sleiman PM, Hu W, Greene R, Wood EM, Bing S, Grossman M, Schellenberg GD, Hatanpaa KJ, Weiner MF, White CL 3rd, Brooks WS, Halliday GM, Kril JJ, Gearing M, Beach TG, Graff-Radford NR, Dickson DW, Rademakers R, Boeve BF, Pickering-Brown SM, Snowden J, van Swieten JC, Heutink P, Seelaar H, Murrell JR, Ghetti B, Spina S, Grafman J, Kaye JA, Woltjer RL, Mesulam M, Bigio E, Lladó A, Miller BL, Alzualde A, Moreno F, Rohrer JD, Mackenzie IR, Feldman $\mathrm{HH}$, Hamilton RL, Cruts M, Engelborghs S, De Deyn PP, Van Broeckhoven C, Bird TD, Cairns NJ, Goate A, Frosch MP, Riederer PF, Bogdanovic N, Lee VM, Trojanowski JQ, Van Deerlin VM. Genetic and clinical features of progranulin-associated frontotemporal lobar degeneration. Arch Neurol 2011; 68: 488-497.

12. Chitramuthu BP, Bennett HPJ, Bateman A. Progranulin: a new avenue towards the understanding and treatment of neurodegenerative disease. Brain 2017; 140: 3081-3104.

13. Cruts M, Gijselinck I, van der Zee J, Engelborghs S, Wils H, Pirici D, Rademakers R, Vandenberghe R, Dermaut B, Martin JJ, van Duijn C, Peeters K, Sciot R, Santens P, De Pooter T, Mattheijssens M, Van den Broeck M, Cuijt I, Vennekens K, De Deyn PP, Kumar-Singh S, Van Broeckhoven C. Null mutations in progran- 
ulin cause ubiquitin-positive frontotemporal dementia linked to chromosome 17q21. Nature 2006; 442: 920-924.

14. Dadas A, Washington J, Diaz-Arrastia R, Janigro D. Biomarkers in traumatic brain injury (TBI): a review. Neuropsychiatr Dis Treat 2018; 14: 2989-3000.

15. Daniel R, Daniels E, He Z, Bateman A. Progranulin (acrogranin/ PC cell-derived growth factor/granulin-epithelin precursor) is expressed in the placenta, epidermis, microvasculature, and brain during murine development. Dev Dyn 2003; 227: 593-599.

16. Daniel R, He Z, Carmichael KP, Halper J, Bateman A. Cellular localization of gene expression for progranulin. J Histochem Cytochem 2000; 48: 999-1009.

17. Diaz-Arrastia R, Shahim P, Sandsmark DK. Molecular biomarkers in the neurological ICU: is there a role? Curr Opin Crit Care 2020; 26: 103-108.

18. Eriksen JL. The enigmatic roles of microglial versus neuronal progranulin in neurological disease. Acta Neuropathol 2010 119: 107-109.

19. Faden Al, Loane DJ. Chronic neurodegeneration after traumatic brain injury: Alzheimer disease, chronic traumatic encephalopathy, or persistent neuroinflammation? Neurotherapeutics 2015; 12: 143-150.

20. Gaddam SS, Buell T, Robertson CS. Systemic manifestations of traumatic brain injury. Handb Clin Neurol 2015; 127: 205-218.

21. Greve MW, Zink BJ. Pathophysiology of traumatic brain injury. Mt Sinai J Med 2009; 76: 97-104.

22. Jawaid A, Rademakers R, Kass JS, Kalkonde Y, Schulz PE. Traumatic brain injury may increase the risk for frontotempora dementia through reduced progranulin. Neurodegener Dis 2009; 6: 219-220.

23. Jeromin A, Bowser R. Biomarkers in neurodegenerative diseas es. Adv Neurobiol 2017; 15: 491-528.

24. Karve IP, Taylor JM, Crack PJ. The contribution of astrocytes and microglia to traumatic brain injury. Br J Pharmacol 2016; 173 692-702.

25. Lasek-Bal A, Jedrzejowska-Szypulka H, Student S, WarszWianecka A, Zareba K, Puz P, Bal W, Pawletko K, LewinKowalik J. The importance of selected markers of inflammation and blood-brain barrier damage for short-term ischemic stroke prognosis. J Physiol Pharmacol 2019; 70: 209-217.

26. Li KW, Ganz AB, Smit AB. Proteomics of neurodegenerative diseases: analysis of human post-mortem brain. J Neurochem 2019; 151: 435-445.

27. Loane DJ, Byrnes KR. Role of microglia in neurotrauma. Neurotherapeutics 2010; 7: 366-377.

28. Maas AIR, Menon DK, Adelson PD, Andelic N, Bell MJ, Belli A, Bragge P, Brazinova A, Büki A, Chesnut RM, Citerio G, Coburn M, Cooper DJ, Crowder AT, Czeiter E, Czosnyka M, Diaz-Arrastia R, Dreier JP, Duhaime AC, Ercole A, van Essen TA, Feigin VL, Gao G, Giacino J, Gonzalez-Lara LE, Gruen RL, Gupta D, Hartings JA, Hill S, Jiang JY, Ketharanathan N, Kompanje EJO, Lanyon L, Laureys S, Lecky F, Levin H, Lingsma HF, Maegele M, Majdan M, Manley G, Marsteller J, Mascia L, McFadyen C, Mondello S, Newcombe V, Palotie A, Parizel PM, Peul W, Piercy J, Polinder S, Puybasset L, Rasmussen TE, Rossaint R, Smielewski P, Söderberg J, Stanworth SJ, Stein MB, von Steinbüchel N, Stewart W, Steyerberg EW, Stocchetti N, Synnot A, Te Ao B, Tenovuo O, Theadom A,
Tibboel D, Videtta W, Wang KKW, Williams WH, Wilson L, Yaffe K; InTBIR Participants and Investigators. Traumatic brain injury: integrated approaches to improve prevention, clinical care, and research. Lancet Neurol 2017; 16: 987-1048.

29. Majdan M, Plancikova D, Brazinova A, Rusnak M, Nieboer D, Feigin V, Maas A. Epidemiology of traumatic brain injuries in Europe: a cross-sectional analysis. Lancet Public Health 2016; 1: e76-e83.

30. Martens LH, Zhang J, Barmada S,, Zhou P, Kamiya S, Sun B, Min SW, Gan L, Finkbeiner S, Huang EJ, Farese RV Jr. Progranulin deficiency promotes neuroinflammation and neuron loss following toxin-induced injury. J Clin Invest 2012; 122: 3955-3959.

31. Matzilevich DA, Rall JM, Moore AN, Grill RJ, Dash PK. High-density microarray analysis of hippocampal gene expression following experimental brain injury. J Neurosci Res 2002; 67: 646-663.

32. Menzel L, Kleber L, Friedrich C, Hummel R, Dangel L, Winter J, Schmitz K, Tegeder I, Schäfer MK. Progranulin protects against exaggerated axonal injury and astrogliosis following traumatic brain injury. Glia 2017; 65: 278-292.

33. Morenas-Rodríguez E, Cervera-Carles L, Vilaplana E, Alcolea D, Carmona-Iragui M, Dols-Icardo O, Ribosa-Nogué R, Muñoz-Llahuna L, Sala I, Belén Sánchez-Saudinós M, Blesa R, Clarimón J, Fortea J, Lleó A. Progranulin protein levels in cerebrospinal fluid in primary neurodegenerative dementias. J Alzheimers Dis 2016; 50: 539-546.

34. Naphade SB, Kigerl KA, Jakeman LB, Kostyk SK, Popovich PG, Kuret J. Progranulin expression is upregulated after spinal contusion in mice. Acta Neuropathol 2010; 119: 123-133.

35. Nicoletto BB, Krolikowski TC, Crispim D, Canani LH. Serum and urinary progranulin in diabetic kidney disease. PLoS One 2016; 11: e0165177.

36. Olczak M, Niderla-Bielińska J, Kwiatkowska M, Samojłowicz D, Tarka S, Wierzba-Bobrowicz T. Tau protein (MAPT) as a possible biochemical marker of traumatic brain injury in postmortem examination. Forensic Sci Int 2017; 280: 1-7.

37. Peeters W, van den Brande R, Polinder S, Brazinova A, Steyerberg EW, Lingsma HF, Maas Al. Epidemiology of traumatic brain injury in Europe. Acta Neurochir (Wien) 2015; 157: 1683-1696.

38. Petkau TL, Neal SJ, Orban PC, MacDonald JL, Hill AM, Lu G, Feldman HH, Mackenzie IR, Leavitt BR. Progranulin expression in the developing and adult murine brain. J Comp Neurol 2010; 518: 3931-3947.

39. Plowman GD, Green JM, Neubauer MG, Buckley SD, McDonald VL, Todaro GJ, Shoyab M. The epithelin precursor encodes two proteins with opposing activities on epithelial cell growth. J Biol Chem 1992; 267: 13073-13078.

40. Prout AJ, Wolf MS, Fink EL. Translating biomarkers from research to clinical use in pediatric neurocritical care: focus on traumatic brain injury and cardiac arrest. Curr Opin Pediatr 2017; 29: 272-279.

41. Raitano S, Ordovàs L, De Muynck L, Guo W, Espuny-Camacho I, Geraerts M, Khurana S, Vanuytsel K, Tóth BI, Voets T, Vandenberghe R, Cathomen T, Van Den Bosch L, Vanderhaeghen P, Van Damme P, Verfaillie CM. Restoration of progranulin expression rescues cortical neuron generation in an induced pluripotent stem cell model of frontotemporal dementia. Stem Cell Reports 2015; 4: 16-24. 
42. Rosso SM, Landweer EJ, Houterman M, Donker Kaat L van Duijn CM, van Swieten JC. Medical and environmental risk factors for sporadic frontotemporal dementia: a retrospective case-control study. J Neurol Neurosurg Psychiatry 2003; 74: 1574-1576.

43. Soukup V, Kalousová M, Capoun O, Sobotka R, Breyl Z, Pešl M Zima T, Hanuš T. Panel of urinary diagnostic markers for noninvasive detection of primary and recurrent urothelial urinary bladder carcinoma. Urol Int 2015; 95: 56-64.

44. Suh HS, Choi N, Tarassishin L, Lee SC. Regulation of progranulin expression in human microglia and proteolysis of progranulin by matrix metalloproteinase-12 (MMP-12). PLoS One 2012; 7 : e35115.

45. Tanaka Y, Matsuwaki T, Yamanouchi K, Nishihara M. Exacerbated inflammatory responses related to activated microglia after traumatic brain injury in progranulin-deficient mice. Neuroscience 2013; 231: 49-60.

46. Taylor CA, Bell JM, Breiding MJ, Xu L. Traumatic brain injuryrelated emergency department visits, hospitalizations, and deaths - United States, 2007 and 2013. MMWR Surveill Summ 2017; 66: 1-16.

47. Tolkatchev D, Malik S, Vinogradova A, Wang P, Chen Z, Xu P, Bennett HP, Bateman A, Ni F. Structure dissection of human progranulin identifies well-folded granulin/epithelin modules with unique functional activities. Protein Sci 2008; 17: 711-724.

48. Van Damme P,Van Hoecke A, Lambrechts D, Vanacker P,BogaertE, van Swieten J, Carmeliet P, Van Den Bosch L, Robberecht W. Progranulin functions as a neurotrophic factor to regulate neurite outgrowth and enhance neuronal survival. J Cell Biol 2008; 181: 37-41.

49. Wang WX, Wilfred BR, Madathil SK, Tang G, Hu Y, Dimayuga J, Stromberg AJ, Huang Q, Saatman KE, Nelson PT. miR-107 regulates granulin/progranulin with implications for traumatic brain injury and neurodegenerative disease. Am J Pathol 2010; 177: 334-345.

50. Wilke C, Gillardon F, Deuschle C, Dubois E, Hobert MA, Müller vom Hagen J, Krüger S, Biskup S, Blauwendraat C, Hruscha M, Kaeser SA, Heutink P, Maetzler W, Synofzik M. Serum levels of progranulin do not reflect cerebrospinal fluid levels in neurodegenerative disease. Curr Alzheimer Res 2016; 13: 654-662.

51. Wilke C, Gillardon F,DeuschleC, Hobert MA, Jansen IE, MetzgerFG, Heutink P, Gasser T, Maetzler W, Blauwendraat C, Synofzik M. Cerebrospinal fluid progranulin, but not serum progranulin, is reduced in GRN-negative frontotemporal dementia. Neurodegener Dis 2017; 17: 83-88.

52. Wu J, Wei L, Wang W, Zhang X, Chen L, Lin C. Diagnostic value of progranulin in patients with lupus nephritis and its correlation with disease activity. Rheumatol Int 2016; 36: 759-767.

53. Xie S, Lu L, Liu L, Bi G, Zheng L. Progranulin and short-term outcome in patients with acute ischaemic stroke. Eur J Neurol 2016; 23: 648-655

54. Xu D, Suenaga N, Edelmann MJ, Fridman R, Muschel RJ, Kessler BM. Novel MMP-9 substrates in cancer cells revealed by a label-free quantitative proteomics approach. Mol Cell Proteomics 2008; 7: 2215-2228.

55. Zetterberg H, Blennow K. Fluid markers of traumatic brain injury. Mol Cell Neurosci 2015; 66: 99-102.
56. Zhang B, Gensel JC. Is neuroinflammation in the injured spinal cord different than in the brain? Examining intrinsic differences between the brain and spinal cord. Exp Neurol 2014; 258: 112-120.

57. Zhu J, Nathan C, Jin W, Sim D, Ashcroft GS, Wahl SM, Lacomis L, Erdjument-Bromage $\mathrm{H}$, Tempst P, Wright CD, Ding A. Conversion of proepithelin to epithelins: roles of SLPI and elastase in host defense and wound repair. Cell 2002; 111: 867-878. 